\title{
Research on Marine Engine Fault Diagnosis based on Vibration Signal Analysis
}

\author{
Tao Ge \\ Tianjin Maritime College, Tianjin 300350, China. \\ 15122530002@163.com
}

\begin{abstract}
With the development of signal analysis and processing technology, the application of vibration signal analysis technology in the ship's fault diagnosis is more and more extensive and mature. The article analyses the diagnosis signal types and characteristics of vibration signal in the ship engine fault diagnosis, and constructs the vibration signal measurement and analysis of the principle of framework, and measure and analyses the combined with DASP system of marine diesel engine cylinder liner under different wear conditions, the analysis results and after assembling cylinder liner wear consistent, which verifies the effectiveness and feasibility of the application in ship engine fault diagnosis analysis of the vibration signal.
\end{abstract}

Keywords: Vibration signal; marine engine; fault; diagnosis.k55230.

\section{Introduction}

To improve the comprehensive utilization efficiency and economic benefit of ship machinery and system, the ship machine fault diagnosis technology can ensure that the ship equipment can accurately obtain the relevant important operating parameters of the ship equipment or system in the normal operation (i.e. without dismantling or large area dismantling), and can determine the location and cause of its failure based on the occurrence of abnormal parameters or characteristics, to early warn the operating trends of equipment and systems, and then can achieve the maintenance of equipment safety, equipment accuracy and state, and it is of great significance to prevent accidents, improve the operation quality, save maintenance costs and avoid the faults caused by crew injury. Therefore, it is significant to study and apply the ship fault diagnosis technology to improve the modern management level and comprehensive benefit of the shipbuilding equipment in the shipping industry.

\section{Vibration Signal Analysis and Diagnosis Technology}

When the classification of equipment or system in the running state, we must grasp the internal state parameters or information of ship equipment, to determine whether the overall or partial is normal and predict the fault and its reason and forecast the development trend of failure.

\subsection{Vibration Signal Classification}

The running state parameters or information of ship equipments are mainly included in the following:

Mechanical information mainly includes force, pressure, torque, speed, current, voltage, vibration, sound, temperature, etc.

Electromagnetic information mainly includes current, voltage, electromagnetic induction density, partial discharge, magnetic conductivity, etc.

Chemical information mainly includes the information of gas, liquid and solid.

At present, the condition monitor of ships and equipment (also known as state monitor) ensures whether the machine working state is normal by obtaining the ship equipment of a single parameter (such as temperature, pressure, vibration, etc.). If the parameters obtained are in the permitted range,

The running state of the equipment is normal, otherwise abnormal. And according to the variation or abnormal degree of parameters, it can determine the maintenance countermeasures, and this method is continuous, simple and easy to implement, and has been widely used in ship equipment monitor and fault diagnosis in practice. 
For mechanical systems, vibration and noise are the most important diagnostic information, which is a kind of property that must be produced in the process of machine operation, and even the most sophisticated machines inevitably produce vibration and noise. Mechanical vibration is a special form of motion, usually reflects the "reciprocating movement" near the static equilibrium position of the equipment or system, namely the object is through its equilibrium position and reciprocating change process. As long as the machine operating, especially marine equipment has more or less vibration, therefore, vibration signal analysis and diagnosis takes a big proportion of various diagnostic methods and is very widely applied. Its theory and measurement technology is relatively mature, which is a very important diagnostic method. This paper focused on the analysis of the failure of ship machine equipment by the diagnostic technique.

\subsection{Vibration Signal Measurement Experimental Principle}

During the process of ship engine fault diagnosis, it mainly experiences the process of fault detection, feature extraction, fault identification, fault trend analysis and fault correction etc.

When the diesel engine of marine is in the operation process, the vibration signal source produced has the characteristic of various excitation sources and impact of vibration frequency. According to the simplicity and sensitivity of measurements, the experiment uses the sensor of vibration acceleration to

Measure the signal of diesel engine vibration.

As shown in Figure 1, it is the schematic diagram of the experimental system of this paper. The experimental system consists of three parts: one is part of the sensor, which is the measurement of the vibration signal and mainly composed of various vibration sensors; the second is data show, which is obtained by using piezoresistive amplifier to tansformate diesel engine running state signal or parameters into voltage or current signal to be displayed; third is the data signal storage, treatment and diagnosis. The experimental object is a 6300ZLCA diesel engine with rated power $551 \mathrm{~kW}$ and Ne $500 \mathrm{r} / \mathrm{min}$.

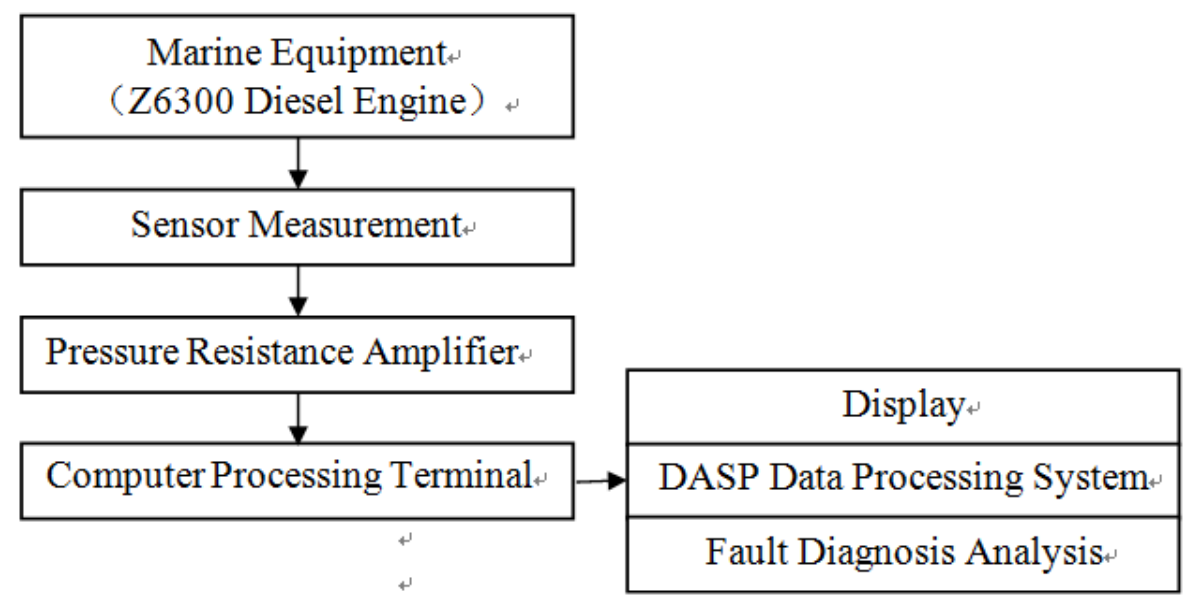

Figure 1 Block Diagram of Experiment System

(1) Sensor Type

There are many kinds of sensors, and has different classification methods, and the commonly used sensors include displacement sensor, temperature sensor, pressure sensor, flow sensor and speed sensor, etc. The performance of the vibration sensor directly affects the effect of vibration measurement, so selecting sensor is based on the actual needs. It needs to compare and choose carefully the main indicators of frequency characteristic, sensitivity, linearity, signal-to-noise ratio, matching effect of output impedance and input impedance of amplifier.

The experimental data of this paper is measured from the 6300ZLCA diesel engine, and the measured signal is the cylinder vibration acceleration. The piezoelectric acceleration sensor has many advantages of small size, high sensitivity, and convenient installation, so the experiment uses Type8614A (X) piezoelectric acceleration vibration sensor produced by KISTLER Company.

(2) Location of Measuring Points Choice 
The gap between the piston and cylinder can cause the diesel engine to produce different vibration signal in the process of operation, and its power spectrum is obviously different, that is, the distribution of vibration energy is obviously different. The purpose of this study is to deduce the operation conditions of wear, etc. through the vibration of cylinder, and provide strong data support for deciding reasonable lifting cylinder time and cycle in engine management, however, the location selection of sensor installation has great impact on measuring of equipment vibration signal, which mainly has the following two conditions: using vibration response of cylinder and body vibration to judge gap change.

When on-site monitoring, sensor cannot be directly installed in the cylinder for the diesel engine structure. Through the transmission characteristics of cylinder and piston impact vibration, transverse vibration of body is mainly caused by the piston impact, and the acceleration response spectrum vibration of cylinder and body are exactly same. Therefore, the sensor is installed on the body, and the measured signal can also reflect the changes of cylinder gap, as shown in Figure 2.

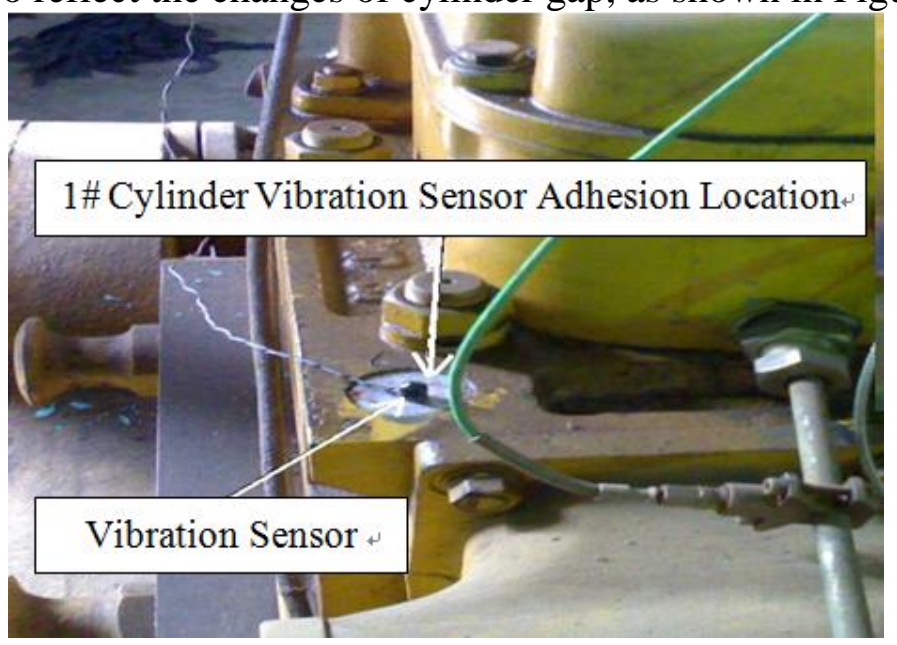

Figure 2 Sensor Installation Location

\subsection{Data Processing System}

This study mainly uses the DASP system for data analysis and processing, which has high resolution, anti-aliasing filter, disk with isolated conditioning channel and flexible user interface etc., and its measurement and display interface as shown in Figure 3.

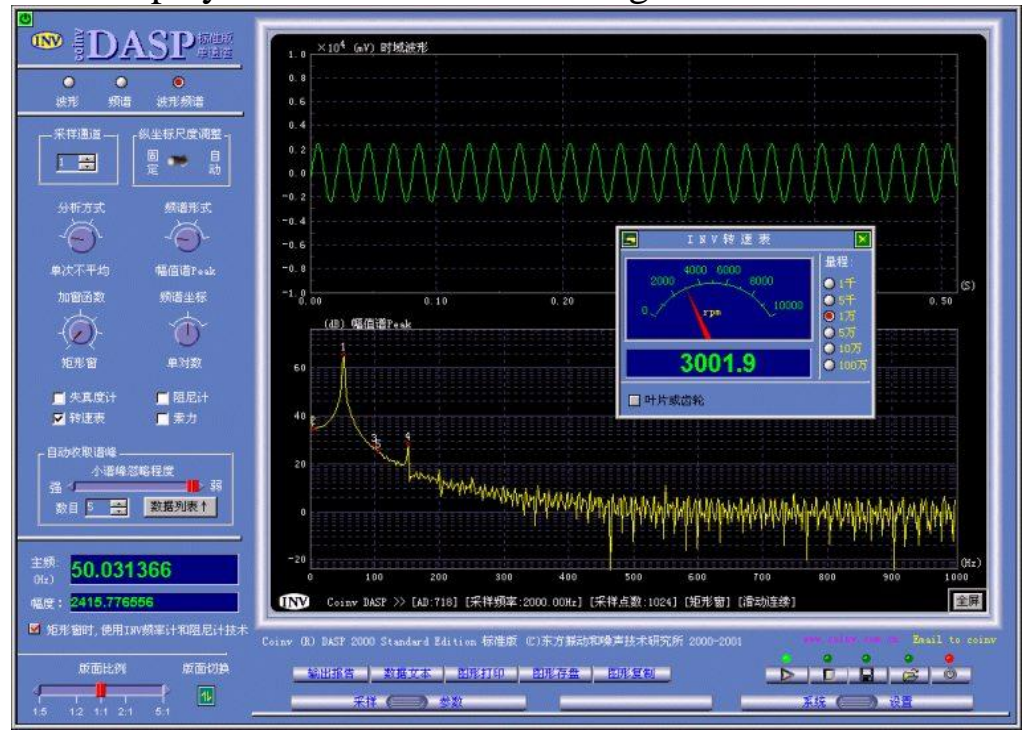

Figure 3 DSAP System Interface

\section{Experimental Data Analysis}

This experiment mainly monitors a different cylinder vibration signal of the diesel engine in the running process by changing the gap between the piston and cylinder. This experiment used three 
different signals of piston and cylinder clearance vibration during $0.07-0.1 \mathrm{~mm}, 0.2-0.25 \mathrm{~mm}$ and $-0.85 \mathrm{~mm} 0.7$ to measure and compare signals. The vibration signal power spectrum after DSAP system processing is as shown in Figure 4 (1), (2), (3). It clearly visible when the gap between piston and cylinder sleeve is decreasing, the vibration energy is relatively small, mainly concentrated in the first frequency band, and with the gap increasing, the vibration signal energy will move to high frequency, mainly concentrated in the second band. Therefore, in the real-time monitoring the operation of diesel engine in the process, when the components of high frequency power spectrum increases significantly, it can be judged as the precursor of scuffing. During the experiment, the test system is shown in Figure 4 (4) is shown. Through the disassembly inspection of diesel engine, it found that wear of diesel engine piston and cylinder is obvious, that is cylinder scoring phenomenon, which also accurately verified the experimental results.
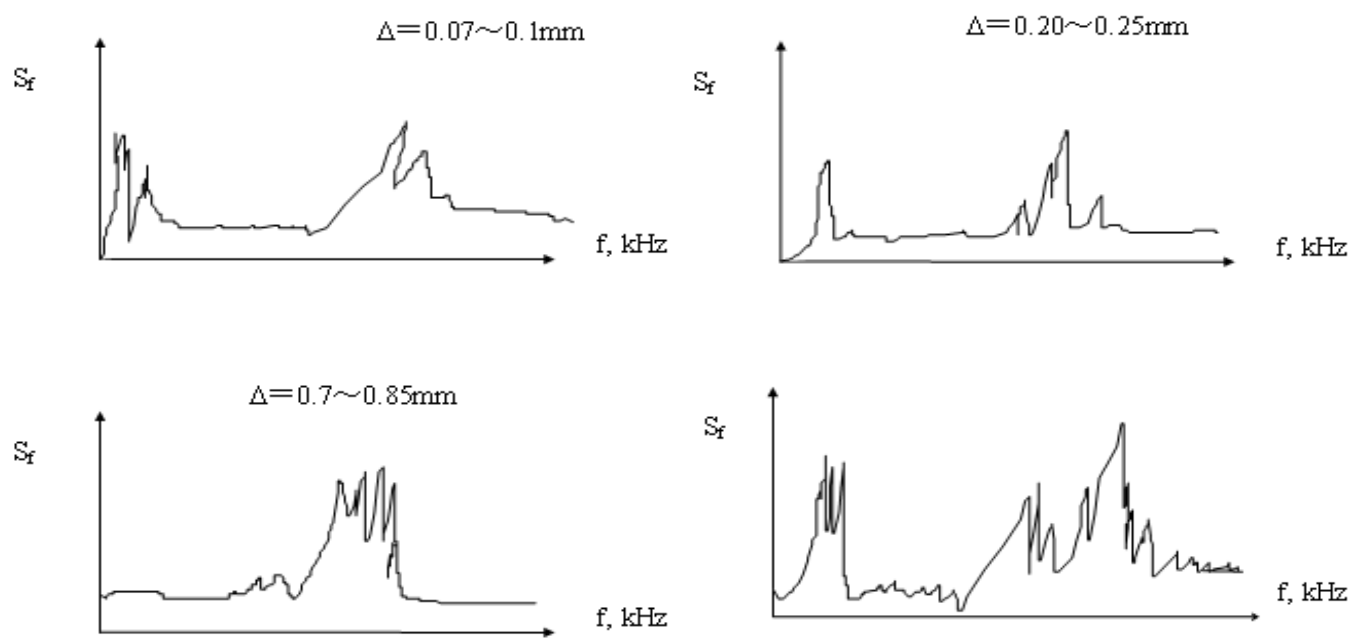

Figure. 4 Vibration Signal Analysis

The experiment has validated the vibration signal analysis has obvious effect and role in the process of marine engine fault diagnosis, as long as it is used in the production process of practice, which has certain positive effect on the safety of shipping and efficient operation.

\section{References}

[1] Qingkai Han, Xiaoguang Yu. The principle and application of modern mechanical fault diagnosis based on vibration analysis. Science Press, 2010, p.25-39.

[2] China Maritime Service Center. Main Propulsion Power Plant. Dalian Maritime University press, 2012, p.212-217.

[3] Ge Tao, Teng Xian-bin, XuYan-ming, et al. Analysis of imbalance vibration signal for crankshaft vibration based on least squares method. Vol. 149 (2014) No. 5, p.61-64.

[4] Bin Li. Marine diesel engine. Dalian Maritime University press, 2013, p. 47-51. 